\title{
The Utility of Scoring Systems in Predicting Early and Late Mortality in Alcoholic Hepatitis: Whose Score Is It Anyway?
}

\author{
Naaventhan Palaniyappan, Venkataraman Subramanian, Vidyasagar Ramappa, \\ Stephen D. Ryder, Philip Kaye, and Guruprasad P. Aithal
}

NIHR Biomedical Research Unit in Gastrointestinal and Liver Diseases, Nottingham University Hospitals NHS Trust and
The University of Nottingham, Nottingham NG7 2UH, UK

Correspondence should be addressed to Naaventhan Palaniyappan, n.palaniyappan@nottingham.ac.uk

Received 14 April 2012; Accepted 26 July 2012

Academic Editor: Neil Guha

Copyright (C 2012 Naaventhan Palaniyappan et al. This is an open access article distributed under the Creative Commons Attribution License, which permits unrestricted use, distribution, and reproduction in any medium, provided the original work is properly cited.

\begin{abstract}
Background. Alcoholic hepatitis (AH) is a distinct clinical entity in the spectrum of alcoholic liver disease with a high shortterm mortality. Several scoring systems are being used to assess the severity of AH but the ability of these scores to predict longterm survival in these patients is largely unknown. Aims. We aim to assess the utility of five different scoring systems Child Pugh (CP), model for end-stage liver disease (MELD), Maddrey's discriminant function (mDF), Glasgow AH score (GAHS), and agebilirubin-INR-creatinine (ABIC) score in predicting shot-term and long-term survival in patients with AH. Methods. Patients with histological evidence of AH were identified from our database. The clinical and biochemical parameters were used to calculate the 5 different scores. The prognostic utility of these scores was determined by generating an ROC curve for survival at 30 days, 90 days, 6 months, and 1 year. Results and Conclusions. All 5 scores with the exception of CP score have a similar accuracy in predicting the short-term prognosis. However, they are uniformly poor in predicting longer-term survival with AUROC not exceeding 0.74 . CP score is a very poor predictor of survival in both short and long term. Abstinence from alcohol was significantly $(P<0.05)$ associated with survival at 1 year.
\end{abstract}

\section{Introduction}

Alcoholic hepatitis $(\mathrm{AH})$ is one of the most recognised "acute on chronic" liver syndromes, wherein patient presents with symptoms and signs of acute decompensation with evidence of chronic liver disease, in the setting of ongoing or recent consumption of excess alcohol [1]. Patients present with progressive jaundice, tender hepatomegaly, and evidence of systemic inflammatory response (SIRS) with characteristic liver biopsy findings of ballooned hepatocytes and Mallory bodies (eosinophilic inclusion bodies) surrounded by neutrophils [2]. $\mathrm{AH}$ is a cause of considerable mortality and morbidity in the Western population. A Danish study reported a 28day mortality rate of $15 \%$ among patients hospitalised for $\mathrm{AH}$ [3]. A pooled one-month mortality of patients with $\mathrm{AH}$ who were treated with placebo in randomised control trials (RCTs) was $22.44 \%$ in US and $18.45 \%$ in Europe [4]. Shortterm prognosis of alcoholic hepatitis is worse than that of decompensated cirrhosis as defined by the system agreed at the Baveno IV consensus conference; 1-year probability of mortality is $20 \%$ in decompensated cirrhosis [5]. Hence, it is important to distinguish patients with $\mathrm{AH}$, in particular those with much worse short-term prognosis, from those with decompensated cirrhosis so that the former group are targeted for specific potentially effective treatments [6-8].

Several scoring systems have been developed and used to assess the severity of $\mathrm{AH}$ and to predict survival in these patients. Maddrey's discriminant function (DF) has been used in clinical practice for more than 30 years [9]. A DF of 32 is used to stratify a patient's severity of $\mathrm{AH}$, patients with a score of $\geq 32$ having a high short-term mortality [10]. Model for end-stage liver disease (MELD) score was initially developed to predict survival in patients with cirrhosis and portal hypertension, but was found to detect short-term survival in patients with AH with good accuracy [11]. However, the cut-off value for MELD score in detecting severe $\mathrm{AH}$ is 
TABLE 1: Histological features in alcoholic hepatitis.

(i) Steatosis

(ii) Ballooned hepatocytes

(iii) Lobular inflammation

(iv) Eosinophilic inclusion bodies-Mallory bodies

(v) Neutrophil infiltration

(vi) Megamitochondria

still controversial with various studies using different values to assess the accuracy of the score. The Glasgow alcoholic hepatitis $(\mathrm{GAH})$ score identifies the subgroup of patients with a DF of $>32$ who will recover without steroids [12]. The ABIC score was developed to categorise patients with AH into high-, moderate-, and low-risk groups based on the risk of death at 90 days and 1 year [13]. The Lille score evaluates the response in serum bilirubin after a 7-day course of corticosteroid therapy and aids the decision in either stopping the corticosteroids or completing a 28-day course [14]. The prognostic value of portal pressure, measured by hepatic venous pressure gradient (HVPG), in chronic liver disease has been well recognised $[15,16]$. However, the influence of portal pressure in $\mathrm{AH}$ as a distinct entity itself has not been established.

The ability of these various scores to predict long-term survival in patients with AH is largely unknown. Therefore, we attempt to assess the utility of the different published scoring systems in predicting short-term (30 and 90 days) and long-term (6 and 12 months) survival in patients with $\mathrm{AH}$.

\section{Methods}

We identified patients with the diagnosis of $\mathrm{AH}$ from our histological database. The decision to refer patients for liver biopsy was made by the primary physician, which was a hepatologist in all cases. As it is our practice to perform liver biopsy through transjugular approach whenever alcoholic hepatitis is suspected, the histological findings, all the transjugular liver biopsies performed in Queens Medical Centre (QMC), Nottingham University Hospitals between 2004 and 2007, were reviewed from the online hospital database. Biopsies showing histological evidence of AH (Table 1) as determined by a single pathologist (PK) were included in the analysis. All the patients undergoing transjugular biopsies have their HVPG measured simultaneously and this was used to identify the degree of portal hypertension in these patients.

The clinical and biochemical parameters for these patients with biopsy-proven $\mathrm{AH}$ was collected from the patient notes and electronic database. These parameters were used to calculate the scoring systems that have been described to guide the management of patients with $\mathrm{AH}$. The measurement units of the biochemical parameters were converted to the relevant units as dictated by the derivation formula of these scores.

Abstinence from alcohol in the short term (3-6 months from the histological diagnosis of $\mathrm{AH}$ ) among these patients
TABLe 2: Patient demographics and histological findings in the 44 biopsy proven alcoholic hepatitis.

\begin{tabular}{lc}
\hline Sex & \\
Male & $25(56.8 \%)$ \\
Female & $19(43.2 \%)$ \\
Mean age, in years & 48 \\
Liver biopsy findings & \\
Steatosis & $42(95.4 \%)$ \\
Necrosis & $10(22.7 \%)$ \\
Neutrophil infiltration & $43(97.7 \%)$ \\
Mallory bodies & $26(59.1 \%)$ \\
Ballooned hepatocytes & $29(65.9 \%)$ \\
Acidophilic bodies & $2(4.5 \%)$ \\
Giant mitochondria & $1(2.27 \%)$ \\
Underlying stage of liver disease & \\
Cirrhosis & $18(40.9 \%)$ \\
\hline
\end{tabular}

TABle 3: Prognostic scores of 44 patients with histological evidence of $\mathrm{AH}$ at the time of biopsy.

\begin{tabular}{lc}
\hline Prognostic scores & N/Median \\
\hline Child-Pugh score $( \pm$ SD) & $10.5( \pm 2.38)$ \\
A $(\%)$ & $5(11.4 \%)$ \\
B $(\%)$ & $8(18.2 \%)$ \\
C $(\%)$ & $31(70.6 \%)$ \\
Model of end-stage liver disease $(\mathrm{MELD})( \pm \mathrm{SD})$ & $18.5( \pm 6.51)$ \\
Maddrey's discriminant factor $(\mathrm{MDF})( \pm \mathrm{SD})$ & $31.6( \pm 19.7)$ \\
Glasgow alcoholic hepatitis $(\mathrm{GAH})$ score $( \pm \mathrm{SD})$ & $7( \pm 1.47)$ \\
ABIC score & $7.19( \pm 1.47)$ \\
HVPG, mmHg $( \pm \mathrm{SD})$ & $13( \pm 6.47)$ \\
\hline
\end{tabular}

was determined during their follow-up clinic appointment or any hospital admissions in this period.

Short-term (30 and 60 days) and long-term (90 days and 1 year) survival was evaluated in these patients. The prognostic value of the scoring systems was determined by generating a receiver operating (ROC) curve and the area under the curve was calculated.

\section{Results}

Over the study period, 140 transjugular liver biopsies were performed in QMC. 44 of these biopsies showed histological evidence of $\mathrm{AH}$. The patient demographics and characteristics of their biopsies are summarised in Table 2. Almost all the biopsies demonstrated steatosis and neutrophil infiltration with over half of them having Mallory bodies and swollen hepatocytes. $40.9 \%(18 / 44)$ of the biopsies showed evidence of underlying cirrhosis.

The clinical and biochemical parameters of these 44 patients with biopsy-proven $\mathrm{AH}$ were used to calculate the various scoring systems that have been proposed (Table 3). The HVPG measurement was not available for a single patient due to malfunctioning instruments during the transjugular liver biopsy. 
TABLE 4: The area under the receiver operating characteristic (AUROC) for prognostic scores for short- and long-term mortality in patients with $\mathrm{AH}$.

\begin{tabular}{lcccccccc}
\hline \multirow{2}{*}{ Prognostic score } & \multicolumn{2}{c}{30 -day mortality } & \multicolumn{2}{c}{ 90-day mortality } & \multicolumn{2}{c}{ 6-month mortality } & \multicolumn{2}{c}{ 1-year mortality } \\
& AUROC & $95 \%$ CI & AUROC & $95 \%$ CI & AUROC & 95\% CI & AUROC & 95\% CI \\
\hline CP & 0.53 & $0.25-0.8$ & 0.47 & $0.21-0.73$ & 0.55 & $0.35-0.76$ & 0.5 & $0.31-0.69$ \\
mDF & 0.79 & $0.64-0.94$ & 0.81 & $0.67-0.95$ & 0.72 & $0.54-0.91$ & 0.63 & $0.43-0.82$ \\
GAHS & 0.78 & $0.54-1$ & 0.81 & $0.61-1$ & 0.73 & $0.54-0.92$ & 0.64 & $0.44-0.84$ \\
ABIC score & 0.74 & $0.46-1$ & 0.79 & $0.55-1$ & 0.67 & $0.44-0.91$ & 0.66 & $0.45-0.87$ \\
MELD & 0.84 & $0.71-0.96$ & 0.85 & $0.74-0.97$ & 0.74 & $0.56-0.92$ & 0.64 & $0.44-0.83$ \\
\hline
\end{tabular}

Follow-up data for one patient was not available as the patient's care was transferred to another hospital. The cumulative 30-day and 90-day mortality in this subgroup was $11.6 \%(5 / 43)$ and $14.0 \%(6 / 43)$. The long-term survival data was unavailable for another patient whose followup was lost. The respective 6-month and 1-year cumulative mortality in this cohort was $21.4 \%(9 / 42)$ and $26.2 \%(11 / 42)$.

In predicting the short-term (30 and 90 day) prognosis in this cohort of patients, GAHS, Maddrey's DF, MELD, and ABIC scores all have a similar accuracy as demonstrated by their AUROC. However, they are uniformly poor in predicting survival beyond 6 months with AUROC not exceeding 0.74 . Childs-Pugh score has been shown to be a very poor predictor of survival in both short and long term (Table 4). Clinically significant portal hypertension (HVPG $\geq 10 \mathrm{mmgHg}$ ) is neither associated with short-term nor long-term prognosis ( $P=$ nonsignificant $)$.

Abstinence from alcohol in 3 to 6 months from the diagnosis of AH was significantly associated with survival at the end of the year $(P<0.05)$ and predicted survival with an AUROC of 0.83 (95 CI: 0.71-0.95).

\section{Discussion and Conclusion}

Despite decades of debate and controversy, the role of histology in identifying the specific cohort described in these studies is still not unequivocally established. In a study that analysed 41 patients biopsied within a month of first presentation with decompensated alcoholic liver disease, none of the histological features were predictive of survival by Cox multivariate analysis [17]. In contrast, a recent study showed that the positive likelihood ratio of the presence of SIRS and clinical features in diagnosing AH is only 1.2, while histological criteria had the best area under the curve in the prediction of adverse outcome [18]. In addition to the lack of consensus, patients with $\mathrm{AH}$ have low platelet count and coagulopathy necessitating transjugular approach for the liver biopsy. As transjugular liver biopsy is available in limited number of centres, algorithms and scores using clinical and simple laboratory parameters are widely used in the clinical management of these patients.

We have described a comparison of 5 different prognostic scores in their value of predicting short- and long-term survival in patients with $\mathrm{AH}$. All the scores with the exception of the CP score have a reasonable accuracy in predicting 30- and 90-day mortality. CP score was originally described to assess the operative risk is patients with established cirrhosis and was developed to predict their survival [19]. The relative ease by which it can be calculated at the bedside has meant that it has remained popular among clinicians. However, its use is limited in predicting prognosis in patients with "acute on chronic liver failure," in particular those with alcoholic hepatitis.

As with CP score, MELD is not a system developed specifically to evaluate AH. MELD score was described initially to predict survival following elective transjugular intrahepatic portosystemic shunts (TIPSS) for the prevention of variceal rebleeding or for the treatment of refractory ascites [20]. Utility of MELD score has since extended to assess the mortality risk in patients with end-stage liver disease and to aid organ allocation priorities in transplant centres [11]. Strength of MELD score is that it functions as a continuous variable and hence the clinical outcome can be accurately estimated based on a particular individual's MELD score. In our cohort, MELD performs well in predicting shortterm outcome in AH. However, estimation of MELD score requires the use of a calculator and there is no consensus on the optimal cut-off value for this score as different studies have chosen different tradeoffs in setting the test threshold (sensitivity and specificity) [21-24].

Value of Maddrey's DF has been verified by more than 30 years experience [10]. Its main use has been in determining the group of patients with $\mathrm{AH}$ that might benefit from corticosteroid therapy. However, Maddrey's DF has often been used to assess the severity of biopsy proven $\mathrm{AH}$ and this has not yet been accepted as a standard practice. The GAH score depends entirely on simple clinical and laboratory parameters and has been shown to have a higher overall accuracy compared to the MELD and Maddrey's DF in predicting in-hospital death [12]. It also stratifies the group of patients with a high Maddrey's DF who will recover without being treated with steroids. The ABIC score was developed in an attempt to risk stratify the death in patients with $\mathrm{AH}$ at 90 days and 1 year [13]. It stratifies patients into high-, moderate-, and low-risk groups. In our study, the performance characteristics of both GAH and ABIC scores were comparable to that of Maddrey's DF. However, the GAH and ABIC scores have not been verified in countries out of which they were derived in.

The influence of portal pressure in the setting of alcoholic hepatitis is not well established. Rincon et al. attempted 
to evaluate the prognostic value of HVPG in patients with acute alcoholic hepatitis and Maddrey's DF of greater than 32 [25]. HVPG of more than $22 \mathrm{mmHg}$ was found to be an independent predictor of in-hospital mortality. Longterm survival was not evaluated in this cohort. In our study, we failed to demonstrate any association between clinically significant portal hypertension (HVPG $\geq 10 \mathrm{mmHg}$ ) with short- and long-term survival.

Our study has demonstrated that majority of the prognostic scores are comparable in their performance characteristics for predicting short-term mortality in patients with $\mathrm{AH}$, and hence, are of similar utility in clinical practice. However, all the scores that we evaluated are uniformly poor in predicting longer-term survival beyond 6 months. We have shown that the abstinence in the first 3 months following the diagnosis of $\mathrm{AH}$ is associated with survival at the end of 1 year. It has been established that abstinence from alcohol is the most important intervention for patients with alcoholic liver disease (ALD). Abstinence has been shown to improve outcome and histological features in all stages of ALD, with improvement noticed in 3 months [26]. As alcohol abstinence is important in improving long-term survival, clinicians should remain focussed in ensuring that these patients have the opportunity to be abstinent from alcohol and continue to do so. The role of pharmacological agents in helping to sustain abstinence is still unclear and requires further investigation.

\section{References}

[1] G. P. Aithal, "Defining "acute on chronic liver failure": an identity crisis!," Indian Journal of Gastroenterology, vol. 29, no. 5, pp. 177-180, 2010.

[2] R. N. M. MacSween and A. D. Burt, "Histologic spectrum of alcoholic liver disease," Seminars in Liver Disease, vol. 6, no. 3, pp. 221-232, 1986.

[3] T. D. Sandahl, P. Jepsen, K. L. Thomsen, and H. Vilstrup, "Incidence and mortality of alcoholic hepatitis in Denmark 1999-2008: a nationwide population based cohort study," Journal of Hepatology, vol. 54, no. 4, pp. 760-764, 2011.

[4] C. H. Yu, C. F. Xu, H. Ye, L. Li, and Y. M. Li, "Early mortality of alcoholic hepatitis: a review of data from placebo-controlled clinical trials," World Journal of Gastroenterology, vol. 16, no. 19, pp. 2435-2439, 2010.

[5] K. M. Fleming, G. P. Aithal, T. R. Card, and J. West, "The rate of decompensation and clinical progression of disease in people with cirrhosis: a cohort study," Alimentary Pharmacology and Therapeutics, vol. 32, no. 11-12, pp. 1343-1350, 2010.

[6] P. Mathurin, J. O'Grady, R. L. Carithers et al., "Corticosteroids improve short-term survival in patients with severe alcoholic hepatitis: meta-analysis of individual patient data," Gut, vol. 60, no. 2, pp. 255-260, 2011.

[7] P. Mathurin, C. Moreno, D. Samuel et al., "Early liver transplantation for severe alcoholic hepatitis," The New England Journal of Medicine, vol. 365, no. 19, pp. 1790-1800, 2011.

[8] E. Nguyen-Khac, T. Thevenot, M.-A. Piquet et al., "Glucocorticoids plus N-Acetylcysteine in severe alcoholic hepatitis," The New England Journal of Medicine, vol. 365, no. 19, pp. 17811789, 2011.
[9] W. C. Maddrey, J. K. Boitnott, and M. S. Bedine, "Corticosteroid therapy of alcoholic hepatitis," Gastroenterology, vol. 75, no. 2, pp. 193-199, 1978.

[10] R. L. Carithers, F. Herlong, A. M. Diehl et al., "Methylprednisolone therapy in patients with severe alcoholic hepatitis. A randomized multicenter trial," Annals of Internal Medicine, vol. 110, no. 9, pp. 685-690, 1989.

[11] P. S. Kamath, R. H. Wiesner, M. Malinchoc et al., "A model to predict survival in patients with end-stage liver disease," Hepatology, vol. 33, no. 2, pp. 464-470, 2001.

[12] E. H. Forrest, C. D. J. Evans, S. Stewart et al., "Analysis of factors predictive of mortality in alcoholic hepatitis and derivation and validation of the Glasgow alcoholic hepatitis score," Gut, vol. 54, no. 8, pp. 1174-1179, 2005.

[13] M. Dominguez, D. Rincón, J. G. Abraldes et al., "A new scoring system for prognostic stratification of patients with alcoholic hepatitis," American Journal of Gastroenterology, vol. 103, no. 11, pp. 2747-2756, 2008.

[14] A. Louvet, S. Naveau, M. Abdelnour et al., "The Lille model: a new tool for therapeutic strategy in patients with severe alcoholic hepatitis treated with steroids," Hepatology, vol. 45, no. 6, pp. 1348-1354, 2007.

[15] J. Bosch and J. C. García-Pagán, "Complications of cirrhosis. I. Portal hypertension," Journal of Hepatology, vol. 32, supplement 1, pp. 141-156, 2000.

[16] C. Ripoll, R. Bañares, D. Rincón et al., "Influence of hepatic venous pressure gradient on the prediction of survival of patients with cirrhosis in the MELD era," Hepatology, vol. 42, no. 4, pp. 793-801, 2005.

[17] D. A. Elphick, A. K. Dube, E. McFarlane, J. Jones, and D. Gleeson, "Spectrum of liver histology in presumed decompensated alcoholic liver disease," American Journal of Gastroenterology, vol. 102, no. 4, pp. 780-788, 2007.

[18] R. P. Mookerjee, C. Lackner, R. Stauber et al., "The role of liver biopsy in the diagnosis and prognosis of patients with acute deterioration of alcoholic cirrhosis," Journal of Hepatology, vol. 55, no. 5, pp. 1103-1111, 2011.

[19] R. N. H. Pugh, I. M. Murray Lyon, and J. L. Dawson, "Transection of the oesophagus for bleeding oesophageal varices," British Journal of Surgery, vol. 60, no. 8, pp. 646-649, 1973.

[20] M. Malinchoc, P. S. Kamath, F. D. Gordon, C. J. Peine, J. Rank, and P. C. J. Ter Borg, "A model to predict poor survival in patients undergoing transjugular intrahepatic portosystemic shunts," Hepatology, vol. 31, no. 4, pp. 864-871, 2000.

[21] M. Sheth, M. Riggs, and T. Patel, "Utility of the Mayo EndStage Liver Disease (MELD) score in assessing prognosis of patients with alcoholic hepatitis," BMC Gastroenterology, vol. 2, article 2, 2002.

[22] W. Srikureja, N. L. Kyulo, B. A. Runyon, and K. Q. Hu, "MELD score is a better prognostic model than Child-TurcottePugh score or Discriminant Function score in patients with alcoholic hepatitis," Journal of Hepatology, vol. 42, no. 5, pp. 700-706, 2005.

[23] A. S. Soultati, S. P. Dourakis, A. Alexopoulou, M. Deutsch, L. Vasilieva, and A. J. Archimandritis, "Predicting utility of a model for end stage liver disease in alcoholic liver disease," World Journal of Gastroenterology, vol. 12, no. 25, pp. 40204025, 2006.

[24] W. Dunn, L. H. Jamil, L. S. Brown et al., "MELD accurately predicts mortality in patients with alcoholic hepatitis," Нераtology, vol. 41, no. 2, pp. 353-358, 2005. 
[25] D. Rincon, O. Lo Iacono, C. Ripoll et al., "Prognostic value of hepatic venous pressure gradient for in-hospital mortality of patients with severe acute alcoholic hepatitis," Alimentary Pharmacology and Therapeutics, vol. 25, no. 7, pp. 841-848, 2007.

[26] B. J. Veldt, F. Lainé, A. Guillygomarc'h et al., "Indication of liver transplantation in severe alcoholic liver cirrhosis: quantitative evaluation and optimal timing," Journal of Hepatology, vol. 36, no. 1, pp. 93-98, 2002. 


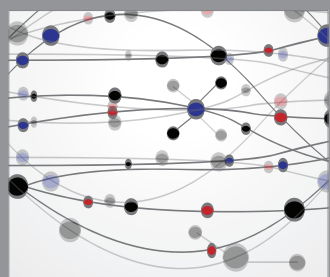

The Scientific World Journal
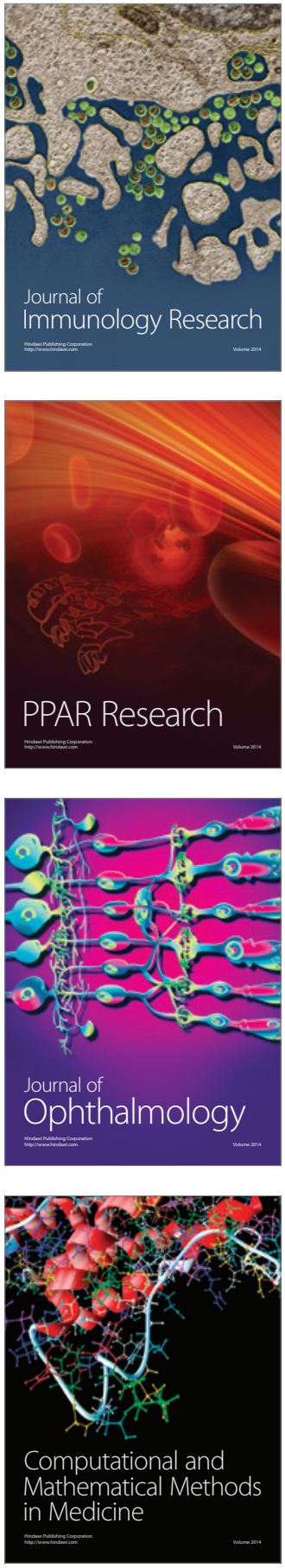

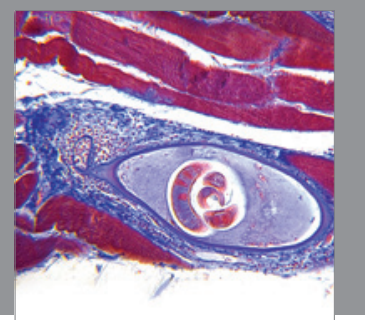

Gastroenterology

Research and Practice
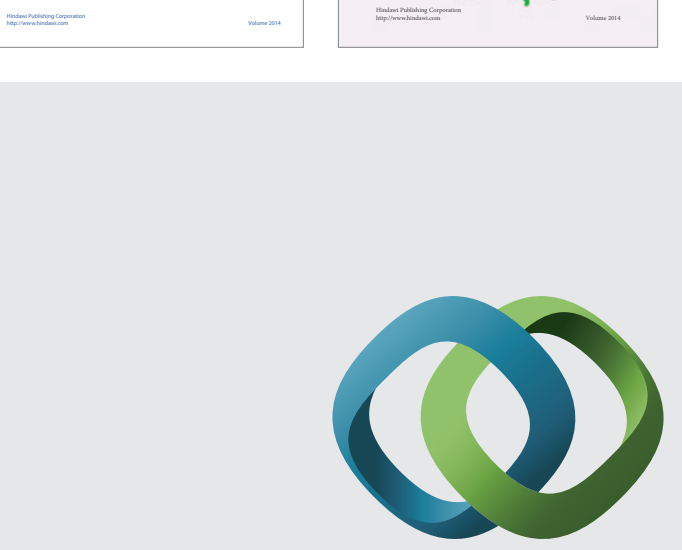

\section{Hindawi}

Submit your manuscripts at

http://www.hindawi.com
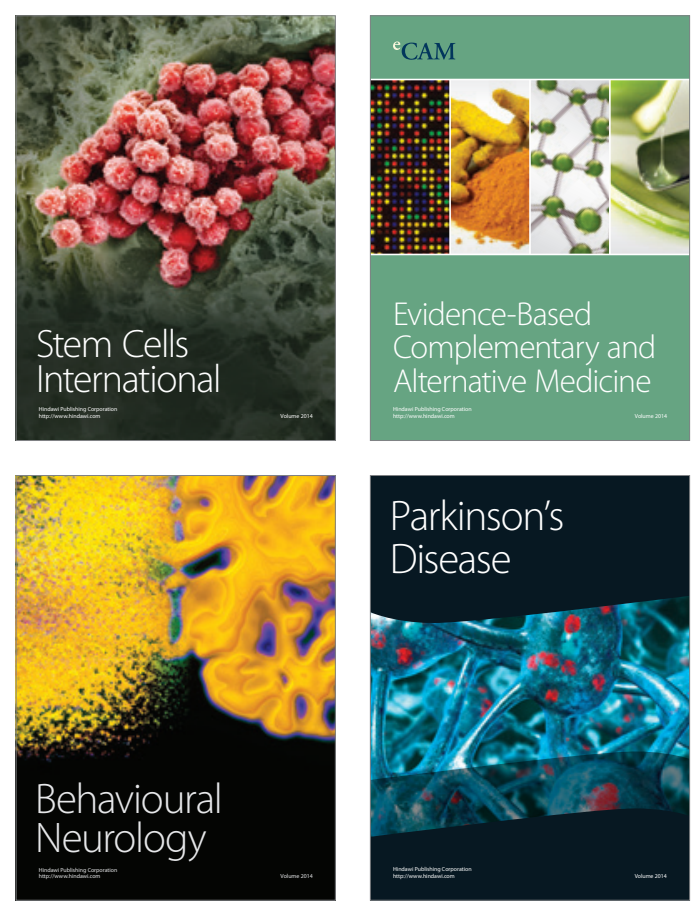

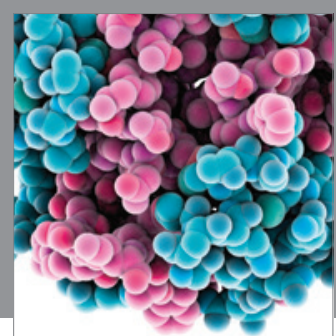

Journal of
Diabetes Research

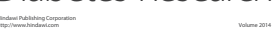

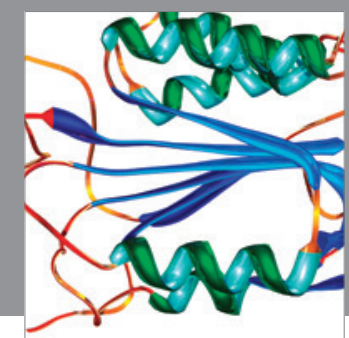

Disease Markers
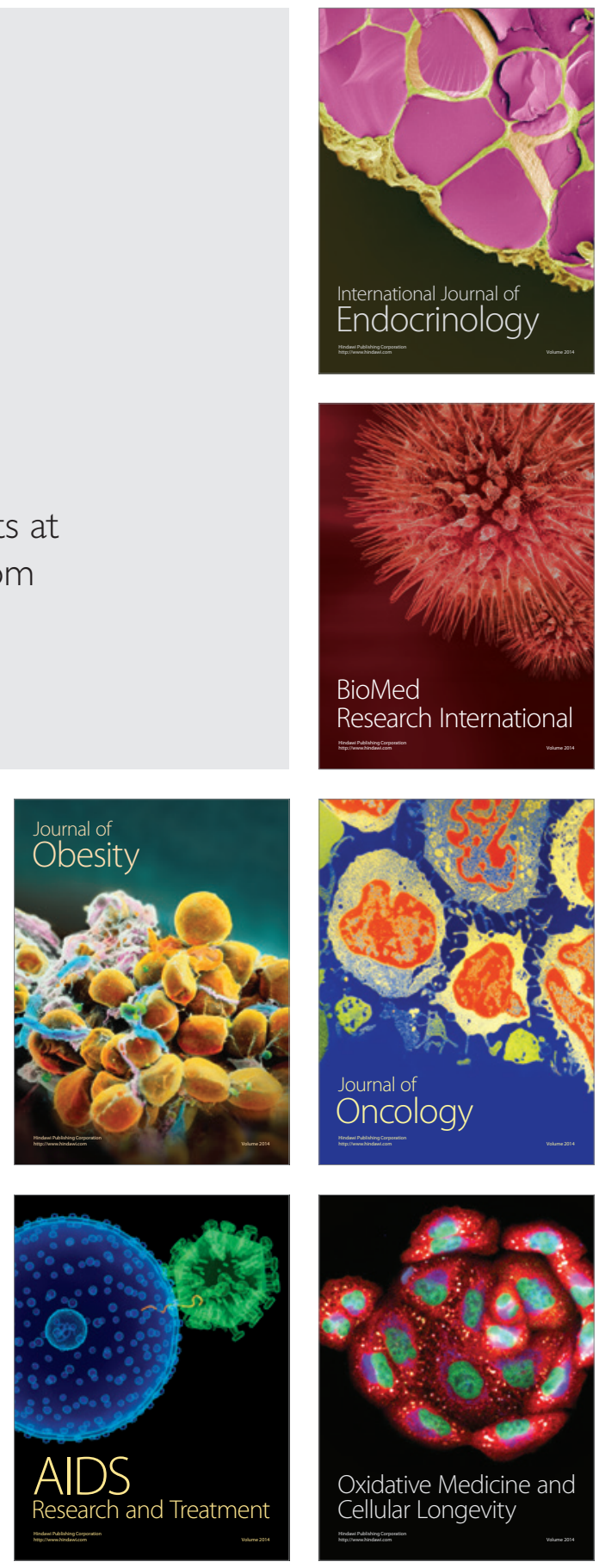